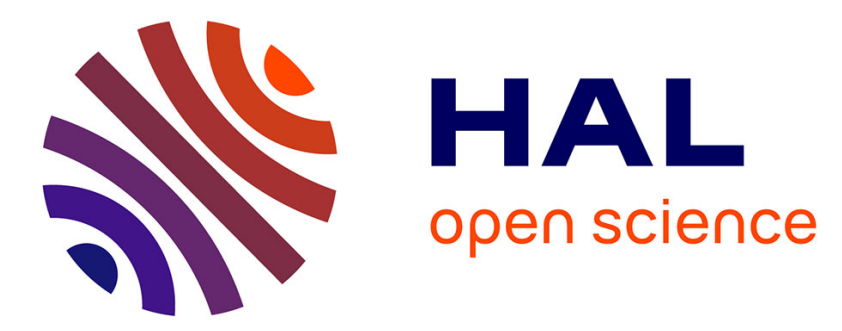

\title{
L'élaboration des choix énergétiques dans les projets urbains, entre apprentissages et négociations. L'exemple des prescriptions immobilières à Bordeaux Saint-Jean Belcier
}

Guilhem Blanchard

\section{To cite this version:}

Guilhem Blanchard. L'élaboration des choix énergétiques dans les projets urbains, entre apprentissages et négociations. L'exemple des prescriptions immobilières à Bordeaux Saint-Jean Belcier. Géographie, Économie, Société, 2017, 19 (2), pp.173-196. 10.3166/ges.19.2017.0009 halshs-01567292

\section{HAL Id: halshs-01567292 \\ https://shs.hal.science/halshs-01567292}

Submitted on 16 Apr 2018

HAL is a multi-disciplinary open access archive for the deposit and dissemination of scientific research documents, whether they are published or not. The documents may come from teaching and research institutions in France or abroad, or from public or private research centers.
L'archive ouverte pluridisciplinaire HAL, est destinée au dépôt et à la diffusion de documents scientifiques de niveau recherche, publiés ou non, émanant des établissements d'enseignement et de recherche français ou étrangers, des laboratoires publics ou privés. 


\title{
L'élaboration des choix énergétiques dans les projets urbains, entre apprentissages et négociations. L'exemple des prescriptions immobilières à Bordeaux Saint-Jean Belcier
}

\section{Energy design in urban development projects, between learning process and negotiations. Insights from Bordeaux, France}

\author{
Guilhem Blanchard*
}

Laboratoire Techniques, Territoires, Sociétés (LATTS - École des Ponts ParisTech, Université ParisEst, CNRS). 6-8 avenue Blaise Pascal, Cité Descartes, 77455 Marne-la-Vallée cedex 2.

\section{*Adresse email : guilhem.blanchard@enpc.fr}

\section{Résumé}

La montée en puissance des politiques publiques de transition énergétique soumet les projets d'aménagement urbain à des pressions croissantes pour faire preuve d'exemplarité énergétique. Documenter la matérialisation des transitions énergétiques urbaines passe par une meilleure compréhension de la façon dont ces pressions sont traduites dans les processus de conception des choix techniques dans les projets d'aménagement. À travers le récit de l'élaboration des prescriptions énergétiques dans un projet bordelais, nous montrons comment ces processus combinent des dynamiques d'apprentissage permettant de construire le cadre cognitif sur lequel s'appuie les acteurs des projets, et des rapports de pouvoir qui focalisent et orientent ces dynamiques. Ce faisant, nous participons à la production de connaissances sur la «petite fabrique » de la ville et des systèmes énergétiques urbains.

\begin{abstract}
The raise of public policies oriented towards energy transition puts increasing pressure on urban development projects, which are more and more supposed to demonstrate exemplarity in energy management. Analyzing the way energy transition is processed into matter then requires
\end{abstract}


a better understanding of how such pressures are translated into the projects' technical design processes. Drawing on the design of energy requirements in one project, we show that the analysis of such processes needs to account both for learning dynamics that build the cognitive framework upon which the actors rely, and for power relations that drive and focus these dynamics. By doing so, we contribute to building a body of knowledge on systems of collective action that embody energy transition into the material fabric of cities and urban energy systems.

Mots-clés : transition énergétique; fabrique urbaine; projet d’aménagement; processus de conception ; apprentissage ; négociation ; Bordeaux Euratlantique.

Keywords: energy transition; urban fabric; urban development project; design process; learning; negotiations; Bordeaux Euratlantique. 


\section{Introduction}

La transition énergétique, entendue comme « une situation émergente fondée sur des actions parfois expérimentales dont l'objet est de faire évoluer le rapport aux ressources et aux modes de gestions de l'énergie» (Roudil, s.d.), vise une reconfiguration radicale des systèmes énergétiques territoriaux suivant des objectifs de sobriété et d'efficacité, de réduction des émissions de gaz à effet de serre et de recours plus important à des ressources renouvelables et de récupération (Jaglin et Verdeil, 2013). Cette transformation se déroule et se joue pour partie dans l'évolution des modes de vie et des pratiques sociales liés à l'énergie (Damon, 2011; Shove, 2010; Subrémon, 2014), et pour partie dans la production de l'environnement construit dans lequel se déroulent ces pratiques. Informer voire accompagner la transition énergétique suppose donc de comprendre comment la fabrique du territoire traduit matériellement les discours et les politiques qui fondent cette transition (Rutherford, 2014). Cette question apparaît d'autant plus importante que les acteurs de cette fabrique considèrent eux-mêmes comme un défi l'intégration des enjeux énergétiques dans leurs projets (Masboungi, 2014).

Partant de ce constat, nous cherchons ici à éclairer les processus de « conception énergétique » dans les opérations d'aménagement urbain, c'est-à-dire à comprendre comment sont élaborés les choix techniques en matière d'énergie dans ces projets. Ces choix concernent essentiellement les performances demandées au bâti (quel objectif de consommation énergétique fixer à chaque projet immobilier, avec quelles obligations pour y parvenir ?) et son approvisionnement en énergie (quelles sources mobiliser, en utilisant quels dispositifs ?). Comprendre leur élaboration consiste à mettre en évidence les acteurs et les dispositifs sociotechniques impliqués, leurs interactions, et les modalités de construction des différentes solutions possibles et d'arbitrage entre elles. Autrement dit, il s'agit d'analyser la mécanique de fabrication des choix d'aménagement, et plus particulièrement des choix en matière d'énergie.

Mais quel prisme adopter pour une telle analyse ? La littérature sur la conception s'articule aujourd'hui autour de deux approches : l'une, plutôt cognitive, tend à accentuer le rôle de la 
connaissance comme base de la conception, avec l'idée sous-jacente qu'une meilleure connaissance permet de meilleures décisions ; l'autre, plutôt stratégiste, investit davantage les rapports de pouvoir et les réseaux qui les sous-tendent pour expliquer les décisions. À la différence d'autres corpus, par exemple sur la gouvernance des projets urbains, les travaux sur les travaux sur l'activité de conception tendent généralement à s'inscrire dans l'une ou l'autre de ces approches ; or, il nous semble plutôt que la compréhension des choix techniques en aménagement combine des processus cognitifs qui ne se traduisent pas directement dans l'action, et des rapports de pouvoir qui ne surdéterminent pas tout. Nous proposons donc ici d'explorer comment s'articulent les lectures cognitive et stratégiste des processus de conception technique des choix énergétiques dans les projets urbains à partir d'une étude de cas: l'élaboration des prescriptions énergétiques dans la ZAC Saint-Jean Belcier, une importante opération d'aménagement de l'agglomération bordelaise. Nous nous appuyons pour ce faire sur des données empiriques approfondies ${ }^{1}$, obtenues dans le cadre d'une recherche doctorale sur les processus de conception des aspects énergétiques à Bordeaux Euratlantique.

L'article est structuré en trois parties. Nous reviendrons dans un premier temps sur l'intérêt des opérations d'aménagement pour l'analyse des transitions énergétiques urbaines: ces projets collectifs sont en effet des espaces d'incubation et de révélation de nouvelles manières de faire la ville face aux enjeux énergétiques. Cependant, à notre sens, la littérature a insuffisamment éclairé la boîte noire des pratiques de conception qui s’y déroulent, notamment parce qu'elle mobilise de façon indépendante les approches cognitive et négociée. Nous proposerons ensuite un récit du processus d'élaboration des prescriptions énergétiques dans la ZAC Saint-Jean Belcier, en mettant en évidence la succession de phases alternativement et parfois simultanément cognitives et négociées. Nous discuterons enfin, à l'aune de ce récit, du cadre conceptuel le plus à même de saisir les choix techniques qui concrétisent la transition énergétique dans la production de l'environnement construit.

\footnotetext{
${ }^{1}$ L'enquête s'appuie ainsi sur une analyse croisée de la documentation interne au projet (rapports d'études, comptes-rendus de réunions, documents d'orientations...) et d'une quarantaine d'entretiens semi-directifs avec ses acteurs. L'anonymat des personnes interrogées et la confidentialité de certaines des données recueillies sont respectés.
} 


\section{Ouvrir la boîte noire des projets urbains pour éclairer l'opérationnalisation de la transition énergétique}

Les villes sont considérées aujourd'hui comme l'une des principales échelles de territorialisation ${ }^{2}$ et d'opérationnalisation de la transition énergétique, à la fois comme acteurs, cibles et instruments privilégiés des politiques en la matière (Emelianoff, 2007; Bulkeley et al., 2010). L'intégration des enjeux de transition énergétique dans la production urbaine a donc fait l'objet d'un effort de recherche important, articulé autour quatre grands types d'objets : les stratégies et politiques territoriales en matière d'énergie et de climat (Emelianoff, 2007, 2014; Chanard, 2011; Coutard et Rutherford, 2010; Poupeau, 2013; Rutherford et Jaglin, 2015), les infrastructures urbaines de l'énergie (Bulkeley et al., 2010; Chevauché et al., 2014; Rutherford et Coutard, 2014), les bâtiments et les projets immobiliers (Guy et Shove, 2000; Guy et Moore, 2005; Camus et al., 2010), et les projets urbains. Ces derniers nous paraissent particulièrement intéressants en ce que leurs acteurs, confrontés à des demandes de performances énergétiques sans précédent, sont amenés à construire collectivement de nouvelles manières de concevoir leurs opérations (1.1.). Pourtant, la littérature n'apporte pas un éclairage suffisant de ces pratiques de conception (1.2.). En particulier, la dualité des approches cognitive et négociée de l'activité de conception nous semble à reconsidérer (1.3.).

\subsection{Les projets urbains, incubateurs et révélateurs des transitions énergétiques urbaines}

| Avant, l'énergie était un élément non pas de choix, mais un élément purement technique : c'est-à-dire qu'il y avait une conception urbaine, éventuellement une conception architecturale, puis on demandait à des bureaux d'études d'intégrer les questions d'énergie, mais dans une vision purement traditionnelle : on mettait de l'électricité, on mettait du gaz, puis après on demandait à EDF et à GDF de venir raccorder et de mettre

\footnotetext{
2 Penser la territorialisation de la transition énergétique revient à porter l'attention sur les différentes échelles et arènes d'action qui contribuent au gouvernement des systèmes énergétiques (Bridge et al., 2013).
} 
les bonnes puissances [...] Le Grenelle de l'Environnement a eu cette vertu de faire en sorte que l'énergie devienne un enjeu stratégique pour une collectivité, pour un aménageur. Tout un tas de raisons font qu'aujourd'hui, on se préoccupe beaucoup plus de cette question en amont des projets d'aménagement. Ça a été une évolution très notable.» (Responsable d'un bureau d'études en énergie et aménagement urbain, avril 2014).

La présence des questions énergétiques dans les opérations d'aménagement urbain n’est pas une nouveauté : l'approvisionnement des bâtiments en chaleur, en eau chaude et en électricité est une préoccupation ancienne, qui suppose d'être prise en compte dans toute opération. Pourtant, jusque récemment, la sphère de l'aménagement ne s'est pas véritablement saisie - en France du moins - de ces questions : elle les externalisait auprès des opérateurs de réseaux (pour la fourniture d'énergie aux bâtiments) et des ingénieurs du bâtiment (pour le dimensionnement des systèmes internes et l'isolation des parois), dont les choix techniques relevaient plutôt de pratiques routinières que d'arbitrages stratégiques propres à chaque projet.

La montée en puissance récente de politiques publiques orientées vers une transition énergétique a cependant engendré des transformations contextuelles importantes pour l'aménagement. Sur le plan réglementaire, les exigences de performance du bâti se durcissent rapidement (réglementations thermiques de 2005, 2012, 2020), tandis que les études de faisabilité pour l'approvisionnement des opérations d'aménagement et des bâtiments en énergies renouvelables sont devenues obligatoires. Au niveau local également, la mise à l'agenda du développement durable et de la transition énergétique a conduit les élus à faire du «développement urbain durable» un élément central de leur politique urbanistique (Emelianoff, 2007). Encouragés par différents appels à projets européens (CONCERTO), nationaux (EcoQuartier) ou régionaux (Nouveaux Quartiers Urbains), les élus n’hésitent pas à exiger des projets urbains l'affichage de performances énergétiques largement supérieures à la réglementation en vigueur. Les opérations d'aménagement sont d'autant plus soumises à ces pressions que leur échelle - qui est celle du quartier - est considérée comme un gisement 
d'amélioration des performances énergétiques sous-exploité et donc stratégique, en particulier par rapport à l'échelle du bâtiment

Pour répondre aux exigences de performances croissantes, les acteurs des projets urbains doivent dorénavant mettre en place des montages techniques, économiques, juridiques et organisationnels complexes, souvent sur-mesure (Souami, 2009), qui supposent d'être pris en compte collectivement très en amont des opérations ${ }^{3}$. Face à un tel défi, les pratiques de conception traditionnelles - qui reposent sur une externalisation des choix énergétiques en fin de processus - sont considérées comme inadaptées (Masboungi, 2014; Terrin, 2014). Certaines méthodologies de prise en compte des questions environnementales dans l'aménagement sont développées (Approche Environnementale de l'Urbanisme, normes ISO...), mais aucune norme stable ne parvient pour l'instant à s'imposer à des projets toujours complexes et singuliers. C'est donc dans chacun des projets locaux que les acteurs bricolent et inventent de nouvelles manières de faire, et l'on peut dire que les opérations d'aménagement sont de ce fait des espaces d'incubation et de révélation des pratiques de fabrique matérielle de la ville, et de la façon dont elles évoluent pour concrétiser les enjeux de transition énergétique des territoires. Pourtant, force est de constater que ces pratiques de conception restent confinées dans une boîte noire que la recherche urbaine n’a pas suffisamment éclairé.

\subsection{La conception (énergétique) des projets urbains : une boîte noire encore insuffisamment éclairée}

On peut distinguer deux périodes dans la production académique et professionnelle sur la conception des projets urbains ${ }^{4}$. La première, qui s'étend de la fin des années 1990 à la fin des années 2000, analyse et accompagne le glissement de l'activité d'aménagement d'une logique de

\footnotetext{
${ }^{3}$ Ces montages peuvent par exemple inclure des mutualisations techniques et/ou des péréquations économiques, ou encore imposer des contraintes en matière de forme urbaine et architecturale, de découpage foncier ou de programmation urbaine.

${ }^{4}$ Nous faisons principalement référence dans cette partie à la production française. Cela s'explique en partie par la forte sensibilité contextuelle de l'aménagement : les manières de faire de l'aménagement sont très différentes entre la France, la Grande-Bretagne et les États-Unis, par exemple. Pour autant, on pourra retrouver les mêmes tendances dans la littérature anglophone en urban planning et urban design.
} 
planification vers une logique de projet (Club des maîtres d'ouvrage d'opérations complexes, 1996; Pinson, 1999; Bourdin, 2001). Les travaux portent alors essentiellement sur la dimension coopérative de la logique de projet et ses conséquences :

- D'une part, sur les professions de la production urbaine, avec une recomposition des métiers, des modes d'exercice des professions établies et des coopérations interprofessionnelles (Evette et Thibault, 2001; Claude, 2006; Biau et Tapie, 2009).

- D'autre part, sur les pratiques de projet, avec le double constat d'un besoin accru de coordination des nouveaux systèmes d'acteurs, assuré notamment par une «maîtrise d'ouvrage urbaine » en cours de construction (Bourdin, 2001; Bonnet, 2005; Frébault, 2005) ; et d'un élargissement de l'activité de conception à l'ensemble de la phase amont des projets, plutôt qu'aux seules conceptions technique et architecturale (Prost, 2003; Arab, 2004; Evette et Terrin, 2006).

Cette première vague de littérature produit de la connaissance sur les systèmes d'acteurs des projets urbains et les interactions qui s'y déroulent. Cependant, à l'exception d'un petit nombre de travaux (Arab, 2004, 2011; Devisme, 2009), l'analyse est peu sensible aux processus de conception technique des choix d'aménagement, qui constituent pourtant la vocation première de l'urbanisme opérationnel (Arab, 2001). En se concentrant sur ce qui pourrait relever d'une gouvernance des projets, les différents travaux évoqués n'éclairent pas suffisamment les processus concret d'élaboration des différentes solutions techniques possibles et d'arbitrage entre elles, pour lesquels les schémas explicatifs ne relèvent pas uniquement de la gouvernance et des dynamiques professionnelles.

La deuxième période que nous souhaitons distinguer ici, plus récente, donne une place plus importante aux choix techniques, notamment autour des thématiques environnementales. On y retrouve deux axes. L'un, qui s'inscrit dans la continuité des travaux précédents, poursuit l'exploration des collectifs de conception des projets urbains en ajoutant aux réseaux d'acteurs 
les dispositifs sociotechniques jouant un rôle dans l'action de conception ${ }^{5}$ (cf. Tardieu, 2015; Debizet, 2016 pour les aspects énergétiques). L'autre porte plutôt sur l'écoconception des projets urbains : il s'agit cette fois d'aider les acteurs de l'aménagement à intégrer au mieux les questions environnementales dans leurs choix, ce qui passe par la production d'outils de modélisation et d'évaluation des choix techniques (Athamena et Belziti, 2013; Peuportier, 2013) et par la diffusion de « bonnes pratiques » pour leur élaboration (Charlot-Valdieu et Outrequin, 2009, 2014; Pinheiro-Croisel, 2013; Beddiar et al., 2015; HESPUL, 2015).

Mais là encore, ces travaux n'ouvrent pas suffisamment la boîte noire de la mécanique des choix d'aménagement : comment les acteurs choisissent-ils, concrètement, les solutions à mettre en place? Comment les interactions entre systèmes d'acteurs, dispositifs sociotechniques, ingénierie de la conception, outils d'aide à la décision, bonnes pratiques... se cristallisent-elles dans l'élaboration des choix techniques ? Et comment saisir ces processus?

\subsection{La conception entre apprentissages et négociations}

Ces questions invitent à mobiliser les travaux sur l'activité de conception dans les projets (urbains, industriels ou autres), qui s'articulent autour de deux grandes approches théoriques. L'une fait plutôt de la conception un ensemble de décisions issues de négociations entre différents acteurs ayant chacun leurs objectifs, leurs moyens d'action, leur stratégie. Inspirée de la sociologie de l'action organisée (Crozier et Friedberg, 1977), cette approche privilégie une lecture de la conception à partir des systèmes d'acteurs qui y prennent part (qui est impliqué dans le jeu de conception, qui en est exclu ?), des rapports de pouvoir qui s'y déroulent (qui a la capacité de faire ou de faire faire quoi, avec quels leviers de négociation ?), et des décisions qui y sont prises (qui fait quels arbitrages, avec quelle capacité normative ?).

L'autre approche, qui puise plutôt son inspiration dans les sciences de gestion, tend à accentuer le rôle de la connaissance et de la coopération comme bases de la conception. Elle s'appuie sur

\footnotetext{
${ }^{5}$ Ces approches s'inspirent de la sociologie des techniques et plus précisément de la sociologie de la traduction (Callon, 1986; Akrich et al., 2006), qui fait l'objet d'un intérêt croissant dans les études urbaines (Farias et Bender, 2010; Rydin, 2012).
} 
un double constat (de Terssac, 1996) : d'une part, la situation de conception est largement inconnue de ses acteurs, qui ne savent pas vraiment quel problème ils cherchent à résoudre, quels résultats ils sont susceptibles de produire, et quels sont leurs intérêts dans le processus ; d'autre part, les savoirs nécessaires à la résolution de la situation sont largement répartis entre les différents acteurs, ce qui rend leur coopération indispensable. La théorie de l'apprentissage organisationnel (Argyris et Schön, 1978; Midler, 2003) fait donc de la conception un processus de construction de connaissances individuelles et collectives sur le projet, auquel chaque acteur contribue par son savoir et ses compétences.

Ces deux approches, l'une plutôt cognitive et l'autre plutôt stratégiste, sont souvent abordées de manière indépendante par la littérature sur les processus de conception. Pourtant, des travaux en science politique (Palier et Surel, 2005) comme en sociologie de la traduction (Latour, 1992; Akrich et al., 2006) montrent tout l'intérêt d'une analyse simultanée des rapports de force et des cadres cognitifs pour embrasser les multiples dimensions de l'action: en effet, dans des contextes marqués par l'incertitude, les intérêts des uns et des autres se construisent au cours de l'action; pour autant, les dynamiques d'apprentissage sont traversées par des logiques d'intérêts qu'elles contribuent d'ailleurs à (re)configurer. Cette double dimension de l'activité de conception apparaît d'ailleurs en filigrane dans les quelques ethnographies disponibles dans la littérature (Kidder, 1981; Bucciarelli, 1994; Yaneva, 2009), tout comme elle est mise en évidence dans les travaux portant sur la gouvernance des et par les projets urbains (Novarina, 1998; Pinson, 2009).

Dès lors, il s'agit de prendre au sérieux l'invitation de ces travaux, qui portent sur d'autres objets que le nôtre, à mobiliser simultanément les approches cognitive et stratégiste pour analyser l'activité de conception des choix techniques de l'aménagement. Nous appuyant sur l'étude d'un cas empirique - l'élaboration des prescriptions énergétiques dans un projet urbain bordelais, nous allons donc examiner la façon dont s'articulent ces deux grilles d'analyse pour expliquer les processus d'élaboration des choix énergétiques d'un projet d'aménagement. 


\section{L'élaboration des prescriptions énergétiques dans la ZAC Saint-Jean Belcier à Bordeaux}

\subsection{Le projet Bordeaux Saint-Jean Belcier et ses aspects énergétiques}

Le projet urbain Bordeaux Saint-Jean Belcier est tout à fait représentatif des « grandes opérations d'aménagement urbain dans des zones délaissées par des activités consommatrices de beaucoup d'espace, [... qui] peuvent attirer les investisseurs et [auxquelles] les responsables politiques locaux attribuent une place centrale dans leur stratégie pour l'agglomération» (Bourdin, 2001, p. 148-149). Situé en péricentre de l'agglomération bordelaise et à proximité immédiate de la gare TGV, le projet s'étend sur une grande superficie (145ha) dont une partie importante est constituée d'espaces industriels et ferroviaires mutables. À la demande des élus locaux qui souhaitent profiter de la garantie de l'État et du portage d'un acteur «tiers » pour mettre en œuvre efficacement ce gros projet intercommunal, l'aménagement est confié à l'Établissement public d'aménagement Bordeaux Euratlantique (ÉPABE), dans le cadre d'une opération d'intérêt national qui s'étend plus largement encore sur les deux rives de la Garonne.

\section{INSERER [FIGURE 1] ICI OU EN VIS-A-VIS DU PARAGRAPHE CI-DESSUS}

Le projet Saint-Jean Belcier, qui prend la forme d'une zone d'aménagement concerté (ZAC), vise à produire un quartier mixte et dense (tableau 1) autour de deux grands objectifs: la constitution d'une nouvelle centralité d'agglomération à partir d'un vaste gisement foncier morcelé et peu attractif ; et le développement d'un centre d'affaires d'envergure nationale voire européenne à destination des activités de services supérieurs et en particulier des grands comptes. Les enjeux prioritaires tournent donc autour de la chaîne de valorisation urbaine (acquisitions foncières, commercialisation, attractivité) plutôt que sur la qualité environnementale du projet. Pour autant, l'État et les collectivités voient dans cette opération, lancée juste après le Grenelle de l'Environnement et le Plan Climat de la ville de Bordeaux, une occasion de déployer une stratégie d'aménagement qui réponde de manière exemplaire aux enjeux du développement urbain durable. 


\section{INSERER [TABLEAU 1] ICI}

La stratégie environnementale de l'opération recouvre quatre axes directement reliés aux questions énergétiques : la politique de mobilité, l’approvisionnement des bâtiments en énergie, les prescriptions faites à chacun des projets immobiliers, et les projets emblématiques ou innovants comme la rénovation de certains îlots, la construction de bâtiments en bois ou le déploiement d'un smart grid. Si ces différents axes sont en interaction, nous nous concentrerons par la suite sur la question des prescriptions énergétiques imposées aux projets immobiliers.

\section{Encadré 1 : Qu'est-ce qu'une prescription énergétique?}

On désigne par prescriptions l'ensemble des préconisations et obligations communiquées par l'aménageur du projet urbain aux maîtres d'ouvrage des projets immobiliers. Elles peuvent être inscrites dans différents documents contractuels et réglementaires (CETE Lyon, 2010): promesses de vente et actes de vente, cahiers des charges de cession de terrains, dossiers de permis de construire. Le non-respect des prescriptions peut, selon les clauses négociées, conduire à la suspension de la vente ou de la délivrance du permis de construire, ou encore au versement d'indemnités à l’aménageur ou à la collectivité.

Les prescriptions visent à s'assurer que chacun des bâtiments construits s'inscrit dans la stratégie globale de l'opération sur les plans urbanistique, paysager, architectural et environnemental. Du point de vue de l'énergie, différents types de prescriptions sont possibles :

- l'obligation de recourir à un procédé constructif ou un système particulier (par exemple : se raccorder à un réseau de chaleur, ou mettre en place une isolation par l'extérieur) ;

- l'imposition d'objectifs de performance par le biais de certifications ou d'indicateurs à respecter (par exemple : faire certifier son bâtiment HQE®, ou imposer une valeur maximale pour l'indicateur de conception bioclimatique du bâtiment) ;

- la justification des choix de conception à l'aide de livrables spécifiques (par exemple : résultats de simulations thermiques dynamiques ou d'analyses de cycle de vie) ; 
- ou encore la mise en place de dispositifs de suivi des performances du bâtiment en activité (par exemple: suivi voire garantie des consommations énergétiques, ou accompagnement et sensibilisation des usagers et des gestionnaires du bâtiment).

\subsection{L'élaboration des prescriptions énergétiques : récit d'un processus en 3 phases}

Nous proposons ci-après un récit du processus d'élaboration des prescriptions énergétiques dans la ZAC Saint-Jean Belcier en trois phases: le cadrage de la question énergétique, qui s'arrête avec le choix d'une assistance à maîtrise d'ouvrage sur l'énergie ; l'élaboration de la stratégie énergétique opérationnelle et de la charte de développement durable de l'aménageur ; et enfin le choix des prescriptions imposées à chacun des projets immobiliers.

\subsubsection{La préfiguration de l'OIN et le choix d'une assistance à maîtrise d'ouvrage: un cadrage de la question énergétique sous forme d'apprentissage collectif}

$\mid \begin{aligned} & \text { "Avant que [...] devienne notre AMO développement durable, on lui avait confié une } \\ & \text { mission dans la mission de préfiguration et sur la base de ce travail, on a établi un } \\ & \text { document d'orientations stratégiques qui est en fait vraiment la base de notre stratégie et } \\ & \text { de nos valeurs. C'étaient les prémices de notre stratégie énergie [...] C'était le moment où } \\ & \text { on était vraiment au jus de cerveau, on était six. À la fin, on a produit le rapport de fin de } \\ & \text { mission. Quand tu regardes ça rétrospectivement, on continue à suivre ses orientations. » } \\ & \text { (Membre de l'équipe de préfiguration de l'OIN, mars 2015). }\end{aligned}$

Notre récit commence en février 2009. Après trois ans d'études sur l'opportunité d'un vaste projet d'aménagement urbain autour de la gare TGV de Bordeaux Saint-Jean, les collectivités locales et l'État se sont mis d'accord sur la constitution d'une opération d'intérêt national (OIN), et donc sur le portage du projet par un Établissement public d'aménagement (EPA). Une mission de préfiguration est alors lancée : elle a pour objectifs de continuer à constituer de la connaissance sur le territoire, de déterminer les modalités de gouvernance du projet, et de proposer des orientations stratégiques pour le développement urbain. Contrairement à d'autres 
sujets (le foncier, la gouvernance), les questions environnementales ne font pas l'objet de négociations avec l'État et les collectivités ; en revanche, elles donnent lieu à une réflexion visant à décliner dans le projet urbain les objectifs et les principes issus du Grenelle de l'Environnement qui s'achève.

L'équipe de préfiguration, composée de sept personnes assistées d'un bureau d'études environnemental, travaille essentiellement sous forme de séances de brainstorming collectif. Ce travail aboutit à deux types de résultats. D'une part, de la connaissance est produite sur les réseaux structurants dans le périmètre de l'opération (électricité, gaz et assainissement, une étude spécifique étant menée pour évaluer le potentiel de récupération de calories sur ce dernier réseau). D’autre part, quelques grands principes stratégiques sont adoptés: en enrichissant la réflexion énergétique, ils contribuent eux aussi à la constitution d'une connaissance partagée sur le projet. Nous en retenons ici quatre, formalisés dans le rapport de fin de mission, qui vont fournir un cadre à la réflexion ultérieure sur l'élaboration des prescriptions énergétiques :

- Le projet urbain vise à créer un nouveau pôle métropolitain attractif, à la fois pour les activités de services supérieurs et pour les familles. Cet objectif se traduit par une priorité donnée au cadre de vie dans les quartiers à créer, et donc à la qualité d'usage des bâtiments plutôt qu'à leur performance technique intrinsèque : le premier des «trois fondamentaux de la durabilité » du projet est ainsi « faire venir et faire rester ».

- Si le projet porte une ambition importante en matière environnementale, dans la lignée du Grenelle, la volonté est cependant affirmée de territorialiser finement cette ambition en constituant « une appellation d'origine contrôlée (AOC) de l'urbanisme durable ». Il ne s'agit donc pas d'appliquer des solutions génériques, mais d'adapter les solutions existantes au contexte bordelais voire d'inventer de nouvelles solutions : il est ainsi fait référence explicitement à un « droit à l'expérimentation ». 
- Cette territorialisation de l'ambition écologique passe notamment par un travail sur les échelles, depuis le bâtiment jusqu'au périmètre du grand projet en passant par l'îlot ou le quartier. Ce travail conduit à réfléchir aux possibilités de mutualisation voire de compensation, pour privilégier une efficience globale du projet plutôt qu'une surperformance de chacun des bâtiments.

- Enfin, l'équipe appelle à tirer parti du climat bordelais et de la proximité de la Garonne pour privilégier une conception passive et bioclimatique des formes urbaines et architecturales plutôt qu'un recours excessif à des systèmes techniques.

\section{INSERER [FIGURE 2] A PEU PRES ICI}

$\mathrm{Au}$ printemps 2010, la mission de préfiguration s'achève et l'Établissement public d'aménagement Bordeaux Euratlantique (ÉPABE) est créé. L'aménageur souhaite alors lancer un appel d'offres pour des missions d'assistance à maîtrise d'ouvrage (AMO) en matière d'énergie. La rédaction des cahiers des charges de ces consultations n'est pas simple : il faut en effet anticiper de façon suffisamment précise les besoins à venir en expertise pour définir les différentes missions pouvant donner lieu à rémunération. La direction de l'ÉPABE a pour cela recours à des consultations informelles : elle s'intéresse aux retours d'expérience sur d'autres projets en mobilisant ses réseaux personnels et professionnels, et assiste à des présentations, par des bureaux d'études, de leur approche de la question énergétique dans les projets urbains. Le cahier des charges pour l'AMO énergie comprend finalement trois missions directement liées à l'élaboration des prescriptions énergétiques: la scénarisation de l'approvisionnement énergétique des bâtiments, d'une part, doit intégrer une réflexion sur les besoins de consommation des bâtiments et donc les performances qui leur sont demandées ; la rédaction des cahiers des charges des performances énergétiques pour chaque projet immobilier, d'autre part, vise à formaliser sous forme contractuelle les prescriptions choisies ; l'avis sur les avantprojets de bâtiments, enfin, doit permettre d'évaluer le respect de ces prescriptions par les opérateurs immobiliers et donc de réajuster les exigences. 
Les consultations sont lancées à l'été 2010, et dix équipes y répondent. L'analyse des offres montre des différences assez marquées dans la façon d'aborder la question de l'énergie et, plus que le prix, c'est la note méthodologique qui fait pencher la balance en faveur du candidat retenu. Ainsi, l'offre retenue interprète les éléments contenus dans le cahier des charges de consultation et dans l'étude de préfiguration de l'OIN pour proposer un cadrage complémentaire des missions liées à l'énergie. Elle insiste en particulier sur deux points, qui seront - eux aussi largement repris par la suite :

- L'offre souligne d'abord qu'il faut interroger les objectifs de performances des bâtiments à l'aune de l'économie générale d'une solution d'approvisionnement mutualisée, en mettant en avant l'incompatibilité potentielle entre des bâtiments trop performants et la mise en place d'un réseau de chaleur dont l'investissement doit être rentabilisé. Le bureau d'études propose ainsi d'élaborer une stratégie énergétique globale de l'OIN, qui construise une «identité énergétique » du projet autour d'objectifs cohérents en matière de performance du bâti et d'approvisionnement en énergies renouvelables et de récupération. Cette nouvelle mission sera ajoutée au marché initial.

- Elle met ensuite en avant deux enjeux importants pour l'opération : d'une part, le maintien d'un bon confort d'été6, notamment au sein des bâtiments de bureaux, dans une zone climatique où les étés sont chauds; d'autre part, le besoin d'un travail sur les performances réelles des bâtiments en complément des exigences théoriques portées par la réglementation et les certifications, dont les premiers retours d'expérience montrent qu'elles se traduisent rarement dans les consommations réelles.

Finalement, cette première phase, qui s'étend du début de l'année 2009 à la fin de l'année 2010, est une phase de cadrage (ou de problématisation), de la question énergétique : en proposant des grands principes stratégiques et en construisant la mission de l'AMO énergie, les acteurs du

\footnotetext{
${ }^{6}$ La notion de confort d'été renvoie à la température ressentie par les usagers d'un bâtiment en période de fortes chaleurs. L'indicateur le plus courant est le nombre d'heures par an où la température ressentie est supérieure à un certain seuil. La conception du bâtiment (inertie thermique, ombrages, renouvellement de l'air...) et les systèmes de rafraichissement voire de climatisation doivent permettre de maintenir suffisamment bas ce nombre d'heures d'inconfort.
} 
projet délimitent de fait le périmètre du problème à résoudre et les principales pistes pour sa résolution. Ce cadrage est d'autant plus important qu'il ne sera jamais vraiment remis en cause dans la suite du projet. Nous observons par ailleurs qu'il s'agit d'un exemple presque idéaltypique d'une conception sous forme d'apprentissage collectif : à ce stade du projet, les acteurs de la conception n'ont pas vraiment défini d'intérêts à défendre, et le mode d'interaction privilégié est la collaboration au sein d'un collectif de conception réduit plutôt qu'une négociation. Le caractère négocié de la conception apparait cependant dans la phase suivante.

\subsubsection{La construction de la stratégie énergétique et de la charte de transformation durable} de l'ÉPABE: une double dynamique d'apprentissage et de négociation

Reprenons notre récit au début de l'année 2011. L'ÉPABE, assisté de son AMO énergie, souhaite construire sa stratégie énergétique. L'élaboration de cette stratégie met en relation deux collectifs de conception. L'un (collectif A) associe la direction technique de l'ÉPABE et son AMO énergie autour des études énergétiques commandées au bureau d'études. L'autre (collectif B) réunit autour de la direction générale de l'ÉPABE sa direction technique, les chargés de projet plutôt «opérationnels » et l'AMO développement durable, dans des ateliers consacrés aux prescriptions environnementales pour l'OIN. L'élaboration d'une stratégie opérationnelle pour les prescriptions énergétiques, qui dure plus d'un an, se fait alors sous forme d'allers-retours entre les deux collectifs de conception, sous la médiation de la direction technique de l'ÉPABE. Dans le collectif A, les échanges permettent à la direction technique de se constituer un savoir sur les questions énergétiques à partir des retours d'expérience proposés par le bureau d'études. Dans le collectif $\mathrm{B}$, ces savoirs sont confrontés à ceux de la direction générale et des directeurs de projet opérationnels quant aux marchés immobiliers. Il s'agit en effet de déterminer le niveau d'acceptabilité des différentes exigences pour les opérateurs immobiliers qui doivent s'installer sur la ZAC :

«On a eu plusieurs séances de travail [...] pour arriver à définir quelles étaient les prescriptions qu'on pouvait demander aux opérateurs immobiliers en fonction des 
différentes typologies d'opérations [...] donc quels objectifs, quels niveaux de performance, quels échanges de preuves pouvaient être transmis de la part des opérateurs, quels engagements contractuels dans le cadre de nos promesses de vente, les questions des pénalités, des compensations,... » (Membre de la direction technique de l'ÉPABE, janvier 2015).

Entre l'intérêt porté par les opérationnels à l'attractivité vis-à-vis des opérateurs immobiliers et le discours environnemental porté par la direction technique, les débats sont parfois animés. Si l'objet des échanges est bien la construction d'une connaissance partagée sur les prescriptions énergétiques acceptables et souhaitables dans le cadre du projet, cette connaissance est l'objet d'une négociation :

"Ça a été assez compliqué de pouvoir faire passer un certain nombre de sujets, d'exigences, de niveaux de performances, de contrôle des opérateurs... ça a été une négociation difficile avec la direction générale, sachant qu'il fallait qu'on arrive à vendre des terrains. » (ibid.)

Prenons l'exemple de l'un des «points durs » de ces négociations : la limitation des besoins de froid. Deux constats sont partagés par les acteurs du projet: d'une part, l'importance de la qualité d'usage des bâtiments qui passe par un bon confort d'été ; d'autre part, la priorité à donner à la conception bioclimatique des bâtiments par rapport à des systèmes techniques énergivores (ici la climatisation). La question est alors de savoir ce qu'il faut exiger des opérateurs immobiliers en matière de rafraichissement des locaux, et deux débats complémentaires émergent : peut-on, au vu du climat bordelais et des techniques de conception des bâtiments, assurer techniquement un rafraichissement suffisant des locaux sans recourir à la climatisation? et si tel est le cas, dans quelle mesure peut-on l'exiger des opérateurs immobiliers?

C'est le directeur général de l'ÉPABE qui, dans ce processus, opère les arbitrages. Si sa capacité et sa légitimité à prendre une décision interne à l'établissement sont indiscutées, il doit 
cependant construire un compromis collectivement acceptable entre les exigences de commercialisation et celles de qualité environnementale. En effet, au-delà du maintien d'un certain équilibre entre les équipes de l'établissement, le débat qui a lieu en interne autour des besoins de froid pourrait tout à fait se dérouler à nouveau dans d'autres scènes de négociation les discussions avec les collectivités locales et avec les promoteurs immobiliers - où les ressources décisionnelles sont plus équitablement distribuées entre les acteurs. De fait, c'est donc à la suite d'un jeu subtil mêlant apprentissage et négociation que la direction générale entérine un compromis : un seuil d'inconfort est défini, des justifications de prise en compte du confort d'été (simulations thermiques dynamiques, notes justificatives des technologies utilisées) seront demandées aux opérateurs immobiliers, le recours à la climatisation devra être limité aux « cas extrêmes » dans les bâtiments de logements.

Cette décision ne peut être comprise comme un simple apprentissage : elle est l'objet d'intenses négociations entre les membres de l'ÉPABE dont l'objectif premier est de faciliter la commercialisation du foncier auprès des opérateurs immobiliers de tertiaire supérieur, et ceux qui portent des objectifs de qualité d'usage et d'exemplarité environnementale. Mais elle ne peut pas non plus être comprise sans prendre en compte sa dimension cognitive : ce sont par exemple la comparaison avec d'autres projets urbains de même type et l'usage de modèles de calculs qui permettent de convaincre de la faisabilité et de l'intérêt d'un rafraichissement passif des bâtiments de logement plutôt que leur climatisation. Il s'agit donc bien là d'un processus décisionnel où les dimensions cognitive et négociée s'entremêlent.

Parallèlement à ces aller-retours entre collectif $\mathrm{A}$ et collectif $\mathrm{B}$ pour définir la stratégie opérationnelle de l'ÉPABE en matière d'énergie, un troisième collectif de conception (collectif C) travaille à l'élaboration de la charte de développement durable de l'OIN, qui inclut les aspects énergétiques. Ce collectif regroupe l'ÉPABE et ses partenaires institutionnels : la Communauté urbaine de Bordeaux (CUB), les municipalités concernées, l'ADEME. Au cours des discussions, un point fait en particulier l'objet de tensions: le fait que l'ÉPABE limite ses exigences de 
performance des bâtiments aux seuils réglementaires ${ }^{7}$. En effet, certains services des collectivités locales voient-là un «dumping environnemental»menaçant d'autres projets urbains en cours : les opérateurs immobiliers, attirés par une exigence énergétique moindre, seraient susceptibles de s'installer dans la ZAC Saint-Jean Belcier plutôt que dans des projets sous maîtrise d'ouvrage directe des collectivités. Or, la prise en charge de l'aménagement de Saint-Jean Belcier par un établissement public d'État n'efface pas complètement les ressources politiques des services locaux: en effet, la gouvernance de l'ÉPABE comme d'autres établissements publics d'aménagement (Pinson et Morel Journel, 2016a) s'appuie sur la cogestion avec les élus locaux, qui signent d'ailleurs les permis de construire au nom de l'État. Le consensus entre l'aménageur et les services des collectivités est donc systématiquement recherché, les éventuels conflits étant portés devant les maires et le président de la CUB.

Face aux contestations, des négociations sont donc menées et aboutissent encore une fois à un compromis : l'exigence de performances globales est maintenue au niveau réglementaire, mais les exigences sur le froid sont durcies (ni les logements, ni les bâtiments tertiaires « innovants » ne pourront recourir à la climatisation). Par ailleurs, un observatoire sera mis en place pour vérifier la bonne application des différentes prescriptions. Là encore, quand bien même il s'agit d'une négociation, la dimension cognitive ne peut être négligée : l'ÉPABE opère en effet un véritable travail de conviction de ses partenaires pour intégrer dans la connaissance partagée le fait qu'il est au moins aussi important et difficile de travailler sur les performances réelles des bâtiments plutôt que de demander un niveau de performances théoriques très ambitieux. La création d'un observatoire a entre autres pour objectif de justifier a posteriori ce postulat.

La deuxième phase évoquée ici se distingue donc de la précédente en ce qu'elle mêle de façon indissociable une dimension cognitive - on continue à produire de la connaissance partagée sur le projet, et les décisions prises s'appuient sur cette connaissance partagée - et une dimension négociée - les acteurs défendent des intérêts identifiables et les choix de conception sont l'objet

\footnotetext{
${ }^{7}$ Rappelons que ce choix s'explique par le cadrage proposé dans la phase précédente, qui met en avant le travail sur les performances réelles et l'échelle du quartier plutôt que sur des exigences théoriques ambitieuses à l'échelle des bâtiments.
} 
de compromis. C'est également le cas de la troisième et dernière phase, que nous allons aborder ci-après : cependant, la dimension de négociation y est particulièrement prégnante.

\subsubsection{La déclinaison des prescriptions énergétiques pour chaque opération immobilière:} une succession de négociations

À la fin de l'année 2012, après deux ans d'allers-retours entre trois collectifs de conception, la stratégie opérationnelle en matière d'énergie et la charte de développement durable de l'ÉPABE sont actées. À ce stade, les prescriptions énergétiques s'articulent entre un socle obligatoire, applicable à tous les projets immobiliers, et un socle négocié au cas par cas. Pour chacune des prescriptions, des livrables attendus de la part des opérateurs immobiliers sont identifiés (figure 3). Cependant, le choix définitif des prescriptions n'est pas encore arrêté : il va faire l'objet d'une succession de négociations pour chacun des projets immobiliers. Les prescriptions énergétiques vont ainsi être abordées par l'ÉPABE et les opérateurs immobiliers au cours de multiples étapes : le choix d'un promoteur, la rédaction de la fiche de lot et de la promesse de vente, le dépôt des avant-projets de bâtiment avant l'instruction des demandes de permis de construire, et enfin la rédaction et la signature de l'acte de vente. Chacune d'entre elles est l'objet d'un réajustement des exigences envers le projet immobilier, qui se négocie entre l'opérateur immobilier, son équipe de maîtrise d'œuvre (l'architecte et ses bureaux d'études), et l'ÉPABE assisté de son AMO énergie.

\section{INSERER [FIGURE 3] ICI OU EN VIS-A-VIS DU PARAGRAPHE CI-DESSUS}

Ce processus se prête très bien à une lecture sous l'angle de l'analyse stratégique : les acteurs en présence - aménageur, opérateur immobilier, architecte, bureaux d'études... - ont des intérêts identifiables, et élaborent chacun une stratégie pour les défendre en fonction des rapports de pouvoir qu'ils identifient. Aussi bien l'aménageur que l'opérateur immobilier ont la capacité d'interrompre le projet immobilier à chacune des phases, mais tous deux ont intérêt à son aboutissement, ce qui les conduit à élaborer des compromis. C'est bien la négociation qui, à chacune des étapes identifiées ici, constitue le mode d'interaction privilégié des acteurs, et les 
rapports de pouvoir permettent d'expliquer une partie des choix : la montée en puissance des prescriptions énergétiques au cours de l'opération, par exemple, s'explique par le gain d'attractivité du marché immobilier local qui permet à l'aménageur de faire jouer la concurrence entre opérateurs immobiliers pour faire passer un certain nombre d'exigences.

Pour autant, là encore, l'approche cognitive vient compléter une lecture stratégiste : chacune des négociations entre aménageur et opérateur immobilier est en effet l'occasion d'un apprentissage croisé, où les acteurs partagent leur savoir sur les dispositifs sociotechniques envisageables pour concilier les intérêts de chacun. L'ÉPABE est ainsi conduit à commander à son AMO énergie des études complémentaires - par exemple sur certaines solutions de rafraichissement des bâtiments, ou sur les dispositifs de garantie des performances réelles - pour être en mesure de proposer aux opérateurs des solutions acceptables ou bien pour évaluer l'intérêt des solutions proposées par ces derniers.

Finalement, les multiples négociations aboutissent à des prescriptions différenciées selon les projets immobiliers. Le jeu des rapports de pouvoir et des rapports de savoir entre l'aménageur, les opérateurs immobiliers et leurs équipes respectives, engendre des ajustements parfois importants par rapport aux socles obligatoires et négociés envisagés dans la stratégie énergétique et la charte de développement durable de l'ÉPABE - il faut d'ailleurs noter que ces ajustements ne se font pas systématiquement «à la baisse», mais conduisent parfois à augmenter le niveau d'exigences.

\section{Articuler apprentissages et négociations pour éclairer la fabrique de la ville et} les transitions énergétique urbaines : quelques remarques conclusives

\subsection{Des processus d'apprentissage et de négociation qui s'articulent de façon dynamique dans l'élaboration des choix techniques}

Du récit que nous avons proposé ressortent trois constats. Nous avons d'abord montré que le processus d'élaboration des prescriptions énergétiques connaît une succession de phases 
alternativement et parfois simultanément cognitives et négociées (tableau 2). Cela nous montre bien qu'une focale unique ne permet pas de comprendre à elle-seule la trajectoire des choix techniques et que là où s'arrête une lecture, l'autre prend le relais. Ainsi, dans notre exemple, on ne peut pas expliquer les prescriptions imposées aux projets immobiliers sans prendre en compte l'apprentissage collectif ayant abouti au cadrage de la problématique énergétique autour d'un certain nombre de principes. Inversement, une approche exclusivement cognitive laisse dans l'ombre une multitude de négociations à différents niveaux (au sein de l'ÉPABE, entre l'ÉPABE et ses partenaires institutionnels, entre l'ÉPABE et les opérateurs immobiliers), alors même qu'elles sont à l'origine d'un certain nombre d'arbitrages quant aux niveaux d'exigences imposés aux projets immobiliers.

\section{INSERER [TABLEAU 2] ICI}

Le deuxième constat que nous souhaitons mettre en évidence est l'évolution du mode d'interaction privilégié au cours du processus étudié : dans notre exemple, plus le projet avance et plus le caractère négocié des interactions est prégnant. Notre hypothèse est que cette montée en puissance des rapports de pouvoir au cours du projet est liée à deux facteurs :

- D'une part, la progression de la connaissance sur le projet : plus on sait, plus on négocie. En effet, le caractère nouveau de l'intégration des questions énergétiques dans les projets urbains fait que les acteurs n'ont pas (ou peu) de cadre préconstruit les incitant à défendre des orientations initiales. C'est donc au cours du projet que ces acteurs construisent des représentations de leurs intérêts ou de leurs préférences, qui les amèneront ensuite à adopter une stratégie et à défendre des positions.

- D'autre part, la progression du degré d'engagement demandé aux acteurs : plus on est contraint, plus on négocie. En effet, avec l'avancement du projet, les prescriptions se font plus précises, formelles et contraignantes (notamment du point de vue juridique) : on passe ainsi de grands principes à des orientations stratégiques puis à une charte, et enfin

à des documents contractuels voire réglementaires. Normatives, les prescriptions 
deviennent des instruments de pouvoir et sont à ce titre l'objet d'attentions plus soutenues de la part des acteurs.

Enfin, le récit nous montre qu'il ne faut pas pour autant adopter un modèle linéaire trop simpliste où l'apprentissage permettrait de dépasser l'incertitude initiale sur la situation de conception et d'aboutir à une situation connue qui serait alors l'objet de "pures négociations ». Le détail des négociations abordées ici met en évidence la subsistance d'une dimension cognitive, y compris dans les dernières étapes du processus d'élaboration des prescriptions. En effet, quand bien même la connaissance sur le projet a considérablement augmenté par rapport aux premières phases, des zones d'ignorance subsistent, notamment parce que le niveau de détail abordé dans les négociations augmente. Les ultimes négociations entre l’ÉPABE et les opérateurs immobiliers sont donc également des espaces de partage de savoirs et de construction d'une connaissance commune.

\subsection{Vers une conceptualisation intégrée de la conception: une activité politique, cognitive et productive}

Le triple constat présenté ci-dessus nous amène à revenir sur la conceptualisation de l'activité de conception en aménagement. Au début de cet article, nous évoquions en effet deux cadres théoriques qui, sans s'affronter, sont généralement mobilisés de manière indépendante. La sociologie de l'action organisée (Crozier et Friedberg, 1977) propose d'appréhender la conception comme un système d'action concret : il s'agit d'un ensemble articulé de jeux où des acteurs « calculateurs » négocient l'action collective à partir de la représentation qu'ils se font de leurs propres intérêts et des stratégies gagnantes à adopter pour favoriser ces intérêts. Le courant de l'apprentissage organisationnel (Argyris et Schön, 1978; Midler, 2003) insiste quant à lui sur le processus de construction des connaissances individuelles et collectives au cours de l'action.

On pourrait donc penser que ces deux approches offrent des lectures divergentes de la conception et qu'«une lecture des dynamiques organisationnelles en termes de négociation 
suppose, en résumant, que les acteurs n'ont pas de difficulté à mettre en relation leur pratique concrète et leur intérêt propre, que les stratégies des uns et des autres sont clairement constituées ex ante et que le problème essentiel se situe dans la confrontation de ces stratégies non convergentes. Au contraire, l'idée d'apprentissage met plutôt l'accent sur la confusion, l'incertitude des objectifs et des représentations des acteurs, quant à leurs intérêts et leurs stratégies » (Midler, 2003, p. 282). La sociologie de l'action organisée serait ainsi remise en cause par l'incertitude des acteurs quant à leurs intérêts, quand l'apprentissage organisationnel proposerait une lecture essentiellement cognitive de l'action au détriment d'une approche par la négociation.

Pourtant, l'étude de cas que nous avons proposée montre que la mise en dialogue des deux cadres théoriques fournit un cadre conceptuel cohérent et fécond. Midler poursuit d'ailleurs ainsi son propos : «La problématique de l'apprentissage organisationnel, approche cognitive des dynamiques sociales, ne néglige évidemment pas l'importance des jeux politiques. Le mot de stratégie, omniprésent en sociologie comme en gestion, serait d'ailleurs celui que l'on utiliserait le plus fréquemment pour désigner ce processus de focalisation du questionnement collectif. La problématique de l'apprentissage inciterait à enrichir cette notion en mettant la question de la production des connaissances au centre de la stratégie, alors qu'elle est souvent considérée comme extérieure et préalable» (p. 283). L’apprentissage organisationnel n'est donc pas incompatible avec l'analyse stratégique, il consiste simplement à porter l'attention sur la production des connaissances sur lesquelles s'appuient les stratégies. Symétriquement, la sociologie de l'action organisée nous semble compatible avec une situation d'incertitude sur les intérêts, ce que confirme d'ailleurs Friedberg (2003, p. 258) : «On ne comprend pas [...] pourquoi une rationalité stratégique aurait besoin d'un agent stable dont les préférences seraient données une fois pour toutes. En effet, pourquoi appeler stratégique un acteur qui ne changerait pas d'identité, et pourquoi restreindre la capacité de calcul à un univers certain ?».

Dès lors, nous défendons une conceptualisation multidimensionnelle de l'activité de conception en aménagement, qui prenne au sérieux simultanément : 
- les dynamiques d'apprentissage qui participent à la construction du cadre cognitif sur lequel s'appuient les acteurs pour agir ;

- et les négociations entre acteurs, qui d'une part focalisent et orientent les dynamiques d'apprentissage, et d'autre part permettent la construction de compromis lorsque des savoirs et/ou des intérêts s'opposent.

S'ajoute à ces deux éléments la dimension productive de l'activité de conception, que nous ne développerons pas ici mais qui mérite d'être évoquée puisque les projets d'aménagement constituent des dispositifs organisationnels dont la finalité est de faire advenir un morceau de ville en respectant un certain nombre d'exigences en termes de qualité, de coûts, de délais, etc.

C'est donc finalement une conceptualisation intégrée de la conception comme activité simultanément politique, cognitive et productive qui nous semble permettre de comprendre finement l'élaboration des choix techniques, et notamment énergétiques, dans les projets d'aménagement. Mais qu'apporte donc l'analyse de cette micro-fabrique de l'aménagement aux études sur la production urbaine et la mise en œuvre de transitions énergétiques territoriales ?

\subsection{Documenter la production de la ville et la matérialisation des transitions énergétiques urbaines à travers la «petite fabrique » de l'aménagement}

Nous avons cherché à éclairer ici les processus d'élaboration des choix énergétiques dans les projets d'aménagement. En nous appuyant sur l'analyse empirique d'un cas concret, nous avons cherché à mettre en évidence l'entremêlement d'éléments cognitifs et stratégiques dans l'activité collective de conception des aspects énergétiques des projets urbains. Mais au-delà de cet objet assez spécifique, il nous semble que la démarche proposée ici peut être prolongée pour éclairer des pans restés obscurs dans les recherches sur la production urbaine d'une part, et sur la matérialisation des transitions énergétiques territoriales d'autre part.

En ce qui concerne la production urbaine, force est de constater avec N. Arab (2007, p. 155) «la faiblesse empirique et théorique des connaissances sur la dynamique et les mécanismes des projets d'aménagement » : rares sont en effet les travaux qui ont cherché à ouvrir la boîte noire de la 
«petite fabrique urbaine» (Devisme, 2009), c'est-à-dire des pratiques et des processus « ordinaires » qui fondent l'élaboration et la mise en œuvre des choix d'aménagement des villes. Or, c'est peut-être à travers cette analyse microsociologique de l'action que l'on peut préciser comment se concrétisent et s'agrègent des dynamiques mises en lumière à plus grande échelle. Pour prendre un exemple, étudier précisément la façon dont les prescriptions immobilières sont construites dans les opérations d'aménagement pourrait permettre de montrer comment s'articulent des dynamiques telles que la financiarisation (Halbert et Attuyer, 2016) et la néolibéralisation (Pinson et Morel Journel, 2016b) de la production urbaine, la circulation des «bonnes pratiques » et des modèles d'aménagement (Devisme et al., 2007; Carriou et Ratouis, 2014; Holden et al., 2015), ou encore l'évolution des savoirs et des métiers de l'urbanisme (Matthey et Fleury, 2016).

L'intérêt d'une telle analyse apparaît de manière tout à fait prégnante lorsqu'il s'agit de documenter la concrétisation d'une forme de transition énergétique dans la matérialité des systèmes urbains. En effet, alors que certains travaux mettent en évidence la diversité des futurs possibles pour l'énergie en ville et la complexité des facteurs à l'œuvre dans les évolutions actuelles (Rutherford, 2014; Debizet, 2016), il paraît indispensable de s'intéresser à la manière dont ces différents facteurs précipitent dans des choix dits «techniques» qui fondent l'infrastructure matérielle des systèmes énergétiques urbains de demain. Autrement dit, ouvrir la boîte noire des choix techniques dans les projets locaux, et notamment les projets urbains, permet d'analyser plus finement l’aménagement énergétique des territoires en train de se faire. 


\section{Références}

Akrich M., Callon M., Latour B., 2006. Sociologie de la traduction. Textes fondateurs. Presses des Mines, Paris.

Arab N., 2011. Projets urbains, conflits sociotechniques et structure des négociations. Le cas de la ligne B du tramway strasbourgeois. In Hamman P. (dir.), Le tramway dans la ville. Le projet urbain négocié à l'aune des déplacements, Presses universitaires de Rennes, 73-89.

Arab N., 2004. L'activité de projet dans l'aménagement urbain. Processus d'élaboration et modes de pilotage. Les cas de la ligne B du tramway strasbourgeois et d'Odysseum à Montpellier. Thèse en aménagement, École nationale des ponts et chaussées, Marne-la-Vallée.

Arab N., 2001. La coproduction des opérations urbaines : coopération et conception. Espaces et sociétés $105-106,57-81$.

Argyris C., Schön D., 1978. Organizational Learning. Addison-Wesley, Reading.

Athamena K., Belziti D., 2013. Environmental assessment methods and tools at the district scale: review and analysis. Présenté à Sustainable procurement in urban regeneration and renovation, Oulu, Finlande.

Beddiar K., Amjahdi M., Lemale J., 2015. Solutions énergétiques dans les écoquartiers. Dunod, Paris.

Biau V., Tapie G. (dir.), 2009. La fabrication de la ville. Métiers et organisations. Parenthèses, Marseille.

Bonnet M. (dir.), 2005. La conduite des projets architecturaux et urbains: tendances d'évolution. La Documentation française, Paris.

Bourdin A. (dir.), 2001. Projet urbain, maîtrise d'ouvrage, commande. Espaces et sociétés 105 106.

Bridge G., Bouzarovski S., Bradshaw M., Eyre N., 2013. Geographies of energy transition: Space, place and the low-carbon economy. Energy Policy 53, 331-340.

Bucciarelli L., 1994. Designing engineers. MIT Press, Cambridge.

Bulkeley H., Castan-Broto V., Hodson M., Marvin S. (dir.), 2010. Cities and low carbon transitions. Routledge, London.

Callon M., 1986. Éléments pour une sociologie de la traduction. La domestication des coquilles Saint-Jacques et des marins-pêcheurs dans la baie de Saint-Brieuc. L'Année Sociologique 36, 169-208.

Camus C., Durand B., Fenker M., Grudet I., Weber B., 2010. Le projet architectural durable négocié. Pratiques, compétences, valeurs. PUCA, Paris.

Carriou C., Ratouis 0. (dir.), 2014. Actualité des modèles urbanistiques. Métropolitiques [En ligne].

CETE Lyon, 2010. Comment assurer la prise en compte de l'environnement dans le cadre d'une ZAC ? MEEDDM, Paris.

Chanard C., 2011. Territoire et énergie : politiques locales, échelles d'intervention et instruments de mobilisation, de connaissance et d'action. Thèse en géographie, Université de FrancheComté, Besançon.

Charlot-Valdieu C., Outrequin P., 2014. Conception, réalisation et évaluation d'un quartier à très basse énergie. Le Moniteur, Paris.

Charlot-Valdieu C., Outrequin P., 2009. L'urbanisme durable: Concevoir un écoquartier. Le Moniteur, Paris.

Chevauché C., Halpern C., Lorrain D. (dir.), 2014. Villes sobres? Réseaux techniques et transitions. Paris, France.

Claude V., 2006. Faire la ville: les métiers de l'urbanisme au XXe siècle. Parenthèses, Marseille. 
Club des maîtres d'ouvrage d'opérations complexes, 1996. L'aménageur urbain face à la crise de la ville. Éditions de l'Aube, La Tour d'Aigues.

Coutard O., Rutherford J., 2010. Energy transition and city-region planning: understanding the spatial politics of systemic change. Technology Analysis and Strategic Management 22(6), 711-727.

Crozier M., Friedberg E., 1977. L'acteur et le système: les contraintes de l'action collective. Seuil, Paris.

Damon J. (dir.), 2011. Villes à vivre: Modes de vie urbains et défis environnementaux. Odile Jacob, Paris.

de Terssac G., 1996. Le travail de conception : de quoi parle-t-on? In de Terssac G., Friedberg E. (dir.), Coopération et Conception, Octarès Éditions, Toulouse, 1-22.

Debizet G., 2016. Scénarios de transition énergétique en ville. Acteurs, régulations, technologies. La Documentation française, Paris.

Devisme L. (dir.), 2009. Nantes, petite et grande fabrique urbaine. Parenthèses, Marseille.

Devisme L., Dumont M., Roy É., 2007. Le jeu des «bonnes pratiques» dans les opérations urbaines, entre normes et fabrique locale. Espaces et sociétés 131, 15-31.

Emelianoff C., 2014. Local Energy Transition and Multilevel Climate Governance: The Contrasted Experiences of Two Pioneer Cities (Hanover, Germany, and Vaxjo, Sweden). Urban Studies 51(7), 1378-1393.

Emelianoff C., 2007. La ville durable: l'hypothèse d'un tournant urbanistique en Europe. L'Information géographique 71(3), 48-65.

Evette T., Terrin J.-J. (dir.), 2006. Projets urbains: expertises, concertation et conception. Éditions de la Villette, Paris.

Evette T., Thibault E. (dir.), 2001. Interprofessionnalité et action collective dans les métiers de la conception urbaine et architecturale. Éditions de la Villette, Paris.

Farias I., Bender T. (dir.), 2010. Urban Assemblages. How Actor-Network Theory Changes Urban Studies. Routledge, New York.

Frébault J. (dir.), 2005. La maîtrise d'ouvrage urbaine. Le Moniteur, Paris.

Guy S., Moore S.A. (dir.), 2005. Sustainable architectures: cultures and natures in Europe and North America. Spon Press, New York.

Guy S., Shove E., 2000. The Sociology of Energy, Buildings and the Environment: Constructing Knowledge, Designing Practice. Routledge, London.

Halbert L., Attuyer K., 2016. The financialisation of urban production: Conditions, mediations and transformations. Urban Studies 53(7), 1347-1361.

HESPUL, 2015. Intégrer l'énergie dans les projets d'aménagement: de l'urbanisme de planification aux projets opérationnels. Le Moniteur, Paris.

Holden M., Li C., Molina A., 2015. The Emergence and Spread of Ecourban Neighbourhoods around the World. Sustainability 7(9), 11418-11437.

Jaglin S., Verdeil E., 2013. Énergie et villes des pays émergents : des transitions en question. Flux $93-94,7-18$.

Kidder T., 1981. The Soul of a New Machine. Little Brown, Boston.

Latour B., 1992. Aramis, ou l'amour des techniques. La Découverte, Paris.

Masboungi A., 2014. L'énergie au cœur du projet urbain. Le Moniteur, Paris.

Matthey L., Fleury A. (dir.), 2016. Débats. Les métiers de la ville. Cybergeo : European Journal of Geography [En ligne]. 
Midler C., 2003. L'apprentissage organisationnel : une dynamique entre cohérence et pertinence des règles? In de Terssac G. (dir.), La théorie de la régulation sociale de Jean-Daniel Reynaud. Débats et prolongements, La Découverte, Paris, 279-288.

Novarina G., 1998. La construction des demandes sociales par le projet d'urbanisme. Annales de la Recherche Urbaine 80-81, 173-179.

Palier B., Surel Y., 2005. Les «trois I » et l'analyse de l'État en action. Revue française de science politique 55, 7-32.

Peuportier B. (dir.), 2013. Éco-conception des ensembles bâtis et des infrastructures. Presses des Mines, Paris.

Pinheiro-Croisel R., 2013. Innovation et éco-conception à l'échelle urbaine : émergence et modèles de pilotage pour un aménagement durable. Thèse en sciences de gestion, École nationale supérieure des mines de Paris.

Pinson G., 2009. Gouverner la ville par le projet. Presses de Sciences-Po, Paris.

Pinson G., 1999. Projets urbains et construction des agglomérations. Echelles fonctionnelles et politiques. Annales de la Recherche Urbaine 82, 130-139.

Pinson G., Morel Journel C., 2016a. Beyond Neoliberal Imposition: State-Local Cooperation and the Blending of Social and Economic Objectives in French Urban Development Corporations. Territory, Politics, Governance 4(2), 173-195.

Pinson G., Morel Journel C., 2016b. The Neoliberal City - Theory, Evidence, Debates. Territory, Politics, Governance 4(2), 137-153.

Poupeau F.-M., 2013. Simples territoires ou actrices de la transition énergétique? Les villes françaises dans la gouvernance multi-niveaux de l'énergie. Urbia - Les cahiers du développement urbain durable 15, 73-90.

Prost R. (dir.), 2003. Projets architecturaux et urbains: mutation des savoirs dans la phase amont. Éditions du PUCA, Paris.

Rutherford J., 2014. The Vicissitudes of Energy and Climate Policy in Stockholm: Politics, Materiality and Transition. Urban Studies 51(7), 1449-1470.

Rutherford J., Coutard O., 2014. Urban Energy Transitions: Places, Processes and Politics of Socio-technical Change. Urban Studies 51(7), 1353-1377.

Rutherford J., Jaglin S., 2015. Urban energy governance: Local actions, capacities and politics. Energy Policy 78, 173-178.

Rydin Y., 2012. Using Actor-Network Theory to understand planning practice: Exploring relationships between actants in regulating low-carbon commercial development. Planning Theory 12(1), 23-45.

Shove E., 2010. Beyond the ABC: climate change policy and theories of social change. Environment and Planning A 42(6), 1273-1285.

Souami T., 2009. Écoquartiers : secrets de fabrication. Analyse critique d'exemples européens. Les Carnets de l'info, Paris.

Subrémon H. (dir.), 2014. Usages de l'énergie dans l'habitat: la transition énergétique vue d'en bas. Flux 96.

Tardieu C., 2015. Transition énergétique dans les projets urbains: conditions de mise en œuvre. Analyse des cas Paris Rive Gauche, Clichy-Batignolles et Paris Nord Est. Thèse en aménagement, Université de Lille 1 / École des ingénieurs de la ville de Paris.

Terrin J.-J., 2014. Le projet du projet. Concevoir la ville contemporaine. Parenthèses, Marseille.

Yaneva A., 2009. The Making of a Building: A Pragmatist Approach to Architecture. Verlag Peter Lang, Oxford. 


\section{Illustrations}

Figure 1. La ZAC Saint-Jean Belcier : un grand projet d'aménagement urbain à vocation métropolitaine en zone péricentrale de l'agglomération bordelaise.

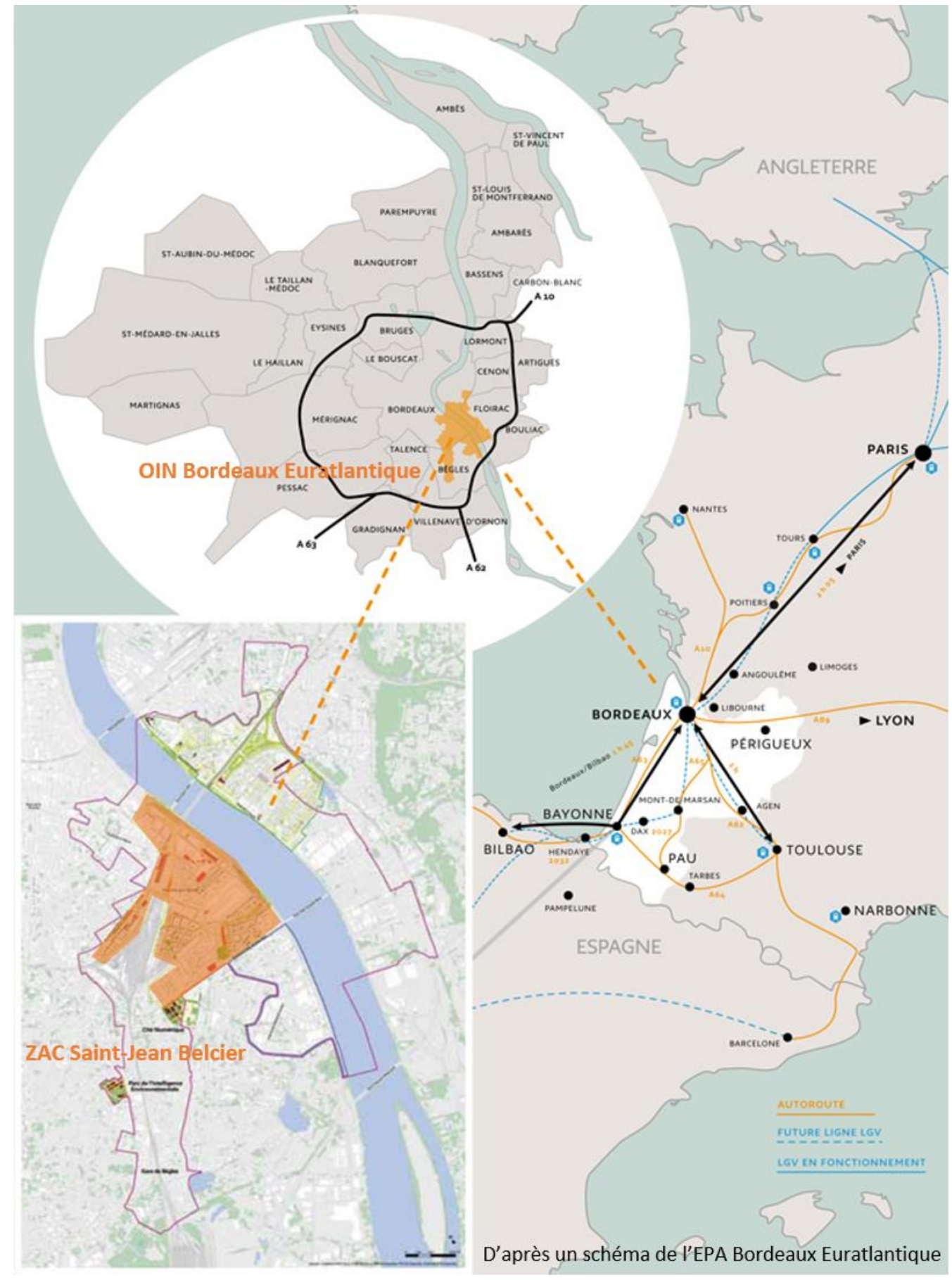


Tableau 1. Caractéristiques prévisionnelles de la ZAC (source : dossier de réalisation, 2013).

\begin{tabular}{|l|l|}
\hline \multirow{5}{*}{ Programmation } & $\begin{array}{l}793000 \mathrm{~m}^{2} \mathrm{SP} \text { sur } 145 \mathrm{ha} \text {, dont } \\
336000 \mathrm{~m}^{2} \text { de logements, } \\
267000 \mathrm{~m}^{2} \text { de bureaux, } \\
\text { et } 190000 \mathrm{~m}^{2} \text { d'autres activités } \\
\text { et équipements }\end{array}$ \\
\hline Phasage & $\begin{array}{l}\text { Phase } 1: 2013-2018 \text { (en violet) } \\
\text { Phase } 2: 2018-2023 \text { (en rouge) } \\
\text { Phase } 3: 2022-2027 \text { (en vert) }\end{array}$ \\
\hline Budget & $\begin{array}{l}\text { Bilan aménageur }: 333 \mathrm{M} € \\
\text { dont 30\% de subventions }\end{array}$ \\
\hline
\end{tabular}


Figure 2. Mutualisation et qualité de vie : deux mots d'ordre dans la réflexion sur les prescriptions énergétiques (source : mission de préfiguration de l'OIN, 2010).

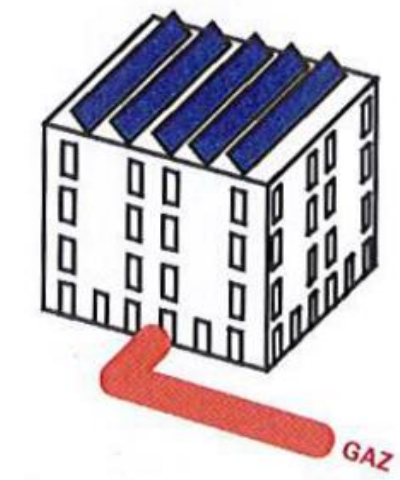

Green (?) building

Compacité

Vitrage $1 / 6$ SHAB

Toiture photovoltaīque

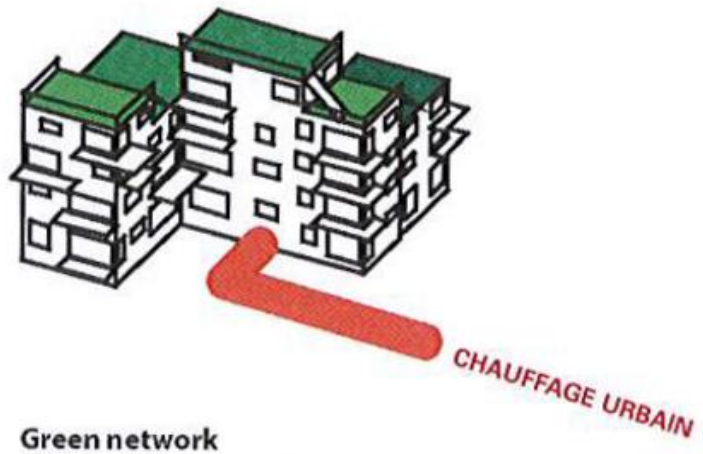

Compacité mais porosité urbaine Façades à plus de $30 \%$ vitrées Grands balcons

Toitures terrasses / espaces collectifs

Un espace extérieur en plus 
Figure 3. Socles de prescriptions en matière de conception bioclimatique et de sobriété énergétique des bâtiments (source : charte de transformation durable, 2013).

\section{Opérateurs immobiliers}

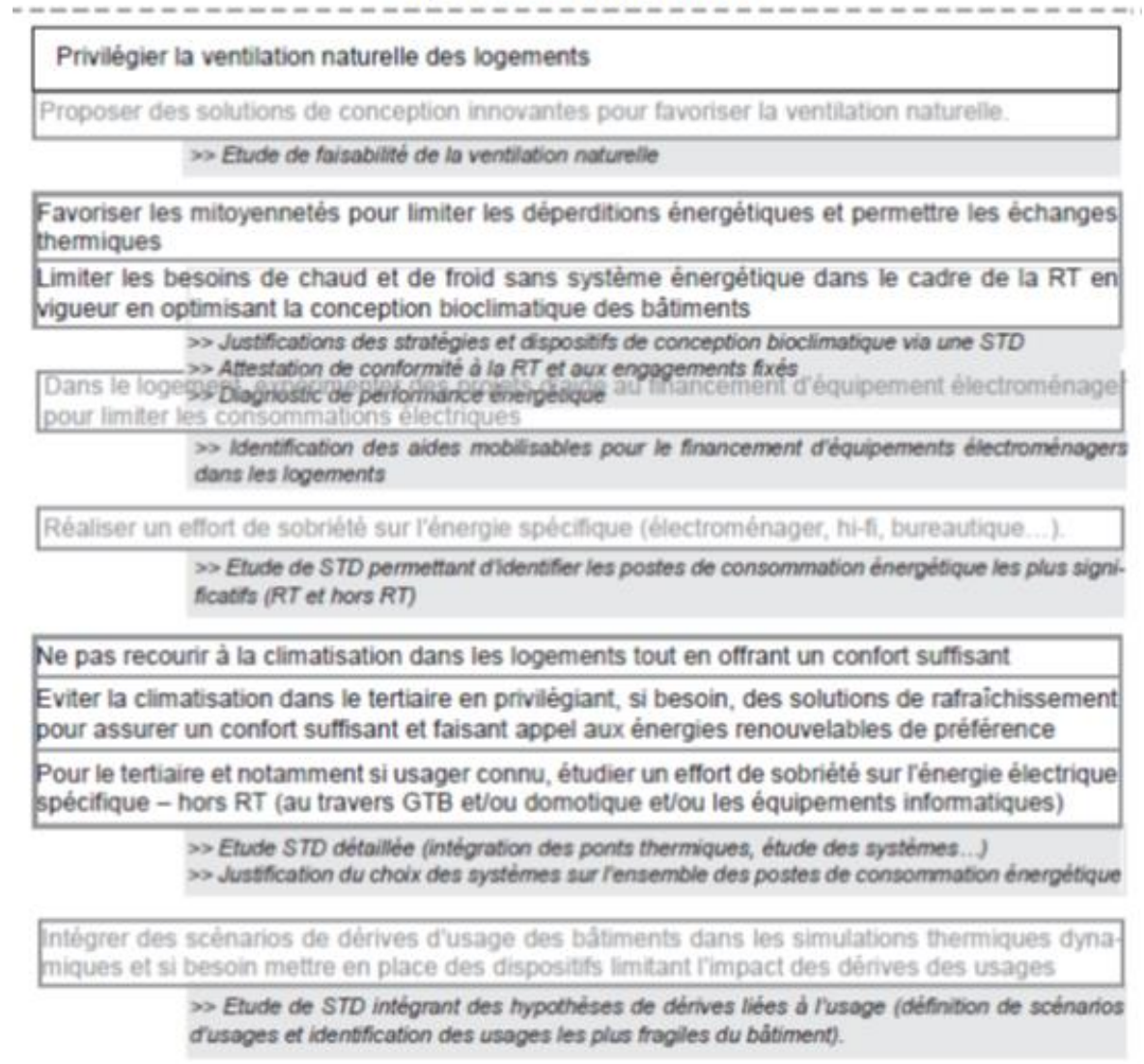


Tableau 2. Les 3 phases d'élaboration des prescriptions énergétiques dans la ZAC Saint-Jean Belcier.

\begin{tabular}{|c|c|c|c|}
\hline & Phase 1 & Phase 2 & Phase 3 \\
\hline Période & $2009-2010$ & 2011-2012 & 2011-2015 \\
\hline Formalisation & $\begin{array}{l}\text { Rapport de fin de } \\
\text { mission de } \\
\text { préfiguration } \\
\text { Cahier des charges du } \\
\text { marché d'AMO-énergie } \\
\text { Offre de l'AMO énergie }\end{array}$ & $\begin{array}{l}\text { Stratégie énergétique } \\
\text { opérationnelle } \\
\text { Charte de } \\
\text { développement durable }\end{array}$ & $\begin{array}{l}\text { Promesses de vente } \\
\text { Dossiers de permis de } \\
\text { construire } \\
\text { Actes de vente }\end{array}$ \\
\hline $\begin{array}{l}\text { Collectif de } \\
\text { conception }\end{array}$ & $\begin{array}{l}\text { Mission de } \\
\text { préfiguration de l'OIN + } \\
\text { AMO développement } \\
\text { durable } \\
\text { Puis ÉPABE + AMO } \\
\text { énergie }\end{array}$ & $\begin{array}{l}\text { Collectif A : direction } \\
\text { technique ÉPABE + } \\
\text { AMO énergie } \\
\text { Collectif B : ÉPABE + } \\
\text { AMO développement } \\
\text { durable } \\
\text { Collectif C : ÉPABE + } \\
\text { Communauté urbaine + } \\
\text { Villes + ADEME }\end{array}$ & $\begin{array}{l}\text { ÉPABE + opérateurs } \\
\text { immobiliers + équipes } \\
\text { de maîtrise d'œuvre } \\
\text { des bâtiments + AMO } \\
\text { énergie }\end{array}$ \\
\hline $\begin{array}{l}\text { Mode d'interaction } \\
\text { privilégié }\end{array}$ & Apprentissage & Mixte & Négociation \\
\hline
\end{tabular}

